\title{
Numerical Modeling for Transmission Dynamics of Hepatitis B Virus Disease
}

\author{
Anmole Razzaq \\ Department of Mathematics \\ NCBA\&E(Gujrat Campus), Pakistan \\ E-mail: anmolrazzaq709@gmail.com
}

\begin{abstract}
Numerical modeling of communicable disease is a device to appreciate the instrument in what way syndrome pushovers and in what way stately. we have studied numerically the dynamics of HBV. We frame an entirely constant Non-Standard Finite Difference (NSFD) structure for a mathematical model of HBV. The introduce numerical array is bounded, dynamically designate and contain the positivity of the solution, which is one of the important requirements when modeling a prevalent contagious. The comparison between the innovative NonStandard Finite Alteration structure, Euler method and Runge-Kutta scheme of order four (RK-4) displays the usefulness of the suggested Non-Standard Finite Alteration scheme. NSFD scheme shows convergence to the exact equilibrium facts of the model for any time steps used but Euler and RK-4 fail for large time steps.
\end{abstract}

Keywords: Hepatitis B Disease, Dynamical System, Numerical Modeling, Convergence.

DOI: $10.7176 / \mathrm{MTM} / 9-1-04$

\section{Introduction}

HBV affects millions of people worldwide each year, where over 40 million cases are reported and kills approximately 600,000 annually. For instance, in Africa it is estimated that annually 400,000 cases happen and an incidence of 50 per 100,000 [5]. The mathematical modeling for transmission dynamics of hepatitis B disease is a capable attitude to grow the conduct of syndrome the population and the foundation, some capable trials of the modeled to the preclude septicity. Dynamical models for the transmission of disease items in a social population, established the Kermack and McKendrick SIR classical epidemic model [1-4], of recommended. These models deliver evaluations for the sequential advancement of infested nodes in a population [5-13]. Now we construct an unreservedly convergent to the numerical model for the transmission dynamics for HBV which conserves all the critical assets of the incessant model [6].

\section{Mathematical Model}

\section{A: Variables and Parameters}

$x(t)$ : Susceptible entities class at time $t$.

$y(t)$ : Infected individuals class at time $t$.

$\mathrm{v}(\mathrm{t})$ : Recover individuals class at time $\mathrm{t}$.

$\lambda$ : Uninfected target cell.

$d_{1}(x)$ : Natural death rate.

$\beta v x$ : Infected target rate.

ay: Death rate. 
$k y$ : Rate of treatment.

$u v$ : Disease induced mortality rate.
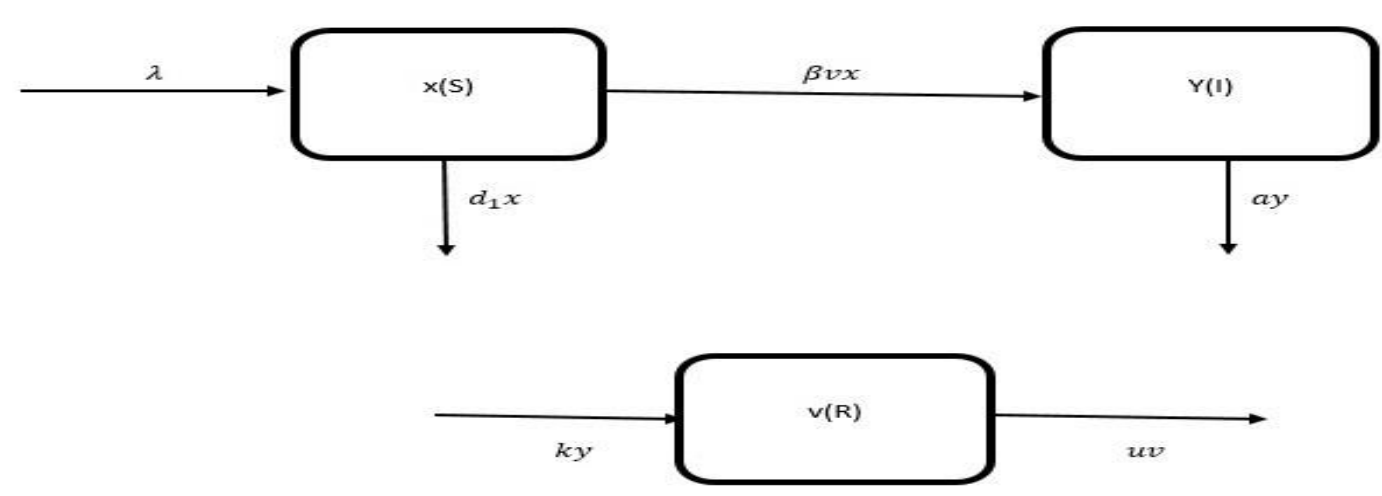

Fig.1 PSIT Hepatitis B Virus Disease Model

The Scheme of Nonlinear Differential Equations(DE) on behalf of the Typical remains specified by:

$$
\begin{aligned}
x^{\prime} & =\lambda-d_{1}-\beta v x \\
y^{\prime} & =\beta v x-a y \\
v^{\prime} & =k y-u v
\end{aligned}
$$

\section{B: Analysis of the Model}

We describe two equilibrium points of system i.e Disease free equilibrium(DFE) and Endemic equilibrium(EE). $\varepsilon_{1}=\left(\frac{\lambda}{d_{1}+\beta v}, 0,0\right)$ and $\quad \varepsilon_{2}=\left(x^{*}, y^{*}, v^{*}\right)$ are stability facts of scheme (1), where

$$
\begin{aligned}
& x^{*}=\frac{\lambda}{d_{1}+\beta v} \\
& y^{*}=\frac{\beta v \lambda}{a\left(d_{1}+\beta \lambda\right)} \\
& v^{*}=\frac{k \beta v \lambda}{a u\left(d_{1}+\beta \lambda\right)}
\end{aligned}
$$

Where $R_{0}=\frac{\theta(\gamma+\mu-\alpha \mu)}{(\gamma+\mu)(\delta+\beta+\mu)}$

$R_{0}$ recognized as Procreative integer who describes the usual number of inferior impurities introduced of the main impurity. $\mathcal{R}_{0}$ is a beginning influence who describe the disease of the exit or persist? If $\mathcal{R}_{0}<1$ then we say that the scheme will observed disease Free Equilibrium (DFE) and iff $\mathcal{R}_{0}>1$ the scheme to involvement Endemic Equilibrium (EE).

\section{Numerical Modeling}

Now we have conferred two standard finite difference structures to unravel the endless dynamical scheme (1) i.e. Euler's Method and Runge-Kutta Method of Order 4.

\section{A: Euler Method}

The Forward Euler's Structure for the unceasing model (1) certain through:

$x^{n+1}=x^{n}+h\left\{\lambda-d_{1}-\beta v x^{n}\right\}$ 


$$
\begin{aligned}
& y^{n+1}=y^{n}+h\left\{\beta v x^{n}-a y^{n}\right\} \\
& v^{n+1}=v^{n}+h\left\{k y^{n}-u v^{n}\right\}
\end{aligned}
$$

\section{B:Numerical Experiments}

Now solve numerical tryouts by expending the values of given parameters Table 1 [6].

Table 1

\begin{tabular}{|c|l|l|}
\hline \multirow{2}{*}{ Parameters } & Values \\
\cline { 2 - 3 } & DFE & EE \\
\hline$d_{1}$ & 0.00379 & 0.8 \\
\hline$a$ & 0.00379 & 0.0044 \\
\hline$u$ & 0.67 & 10 \\
\hline$R_{0}$ & 9 & 0.1 \\
\hline$m$ & 0 & 0.005 \\
\hline$n$ & 0.982 & 0.9 \\
\hline
\end{tabular}

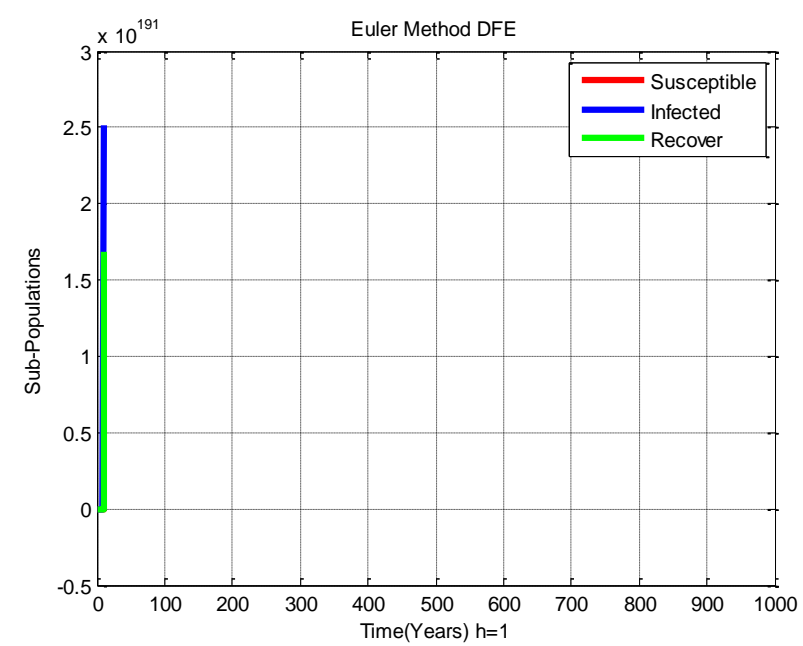

Fig. 2 Euler Method (DFE), $h=1$

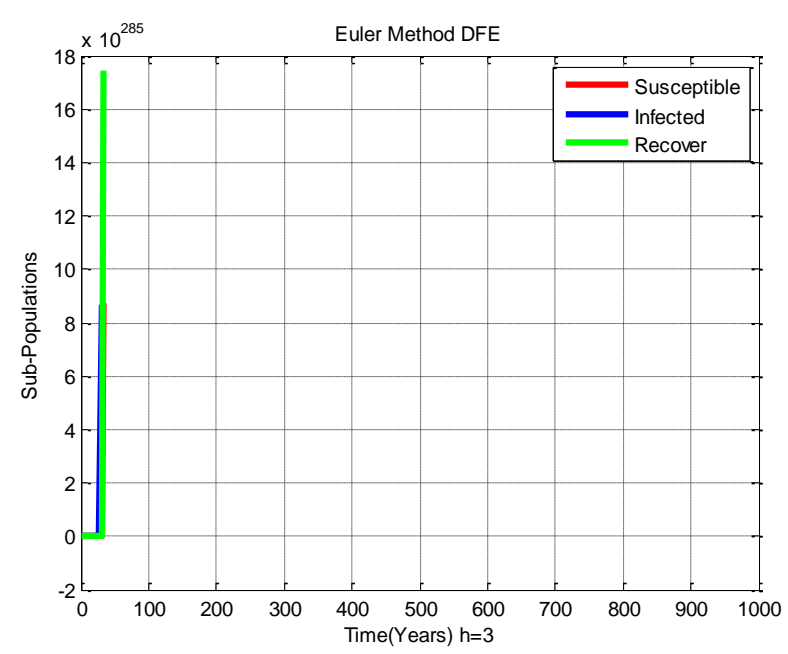

Fig.3 Euler Method (DFE), $h=3$ 


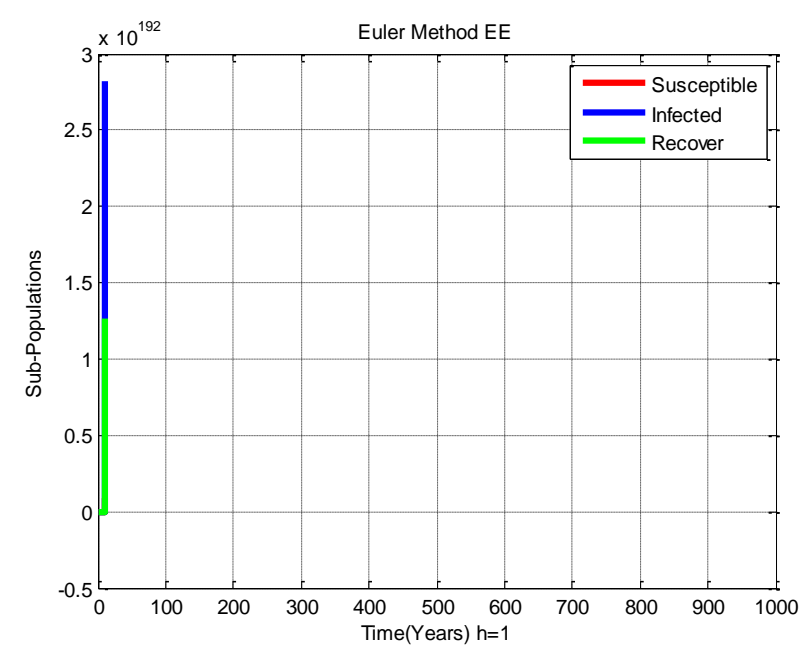

Fig. 4 Euler Method (EE), $h=1$

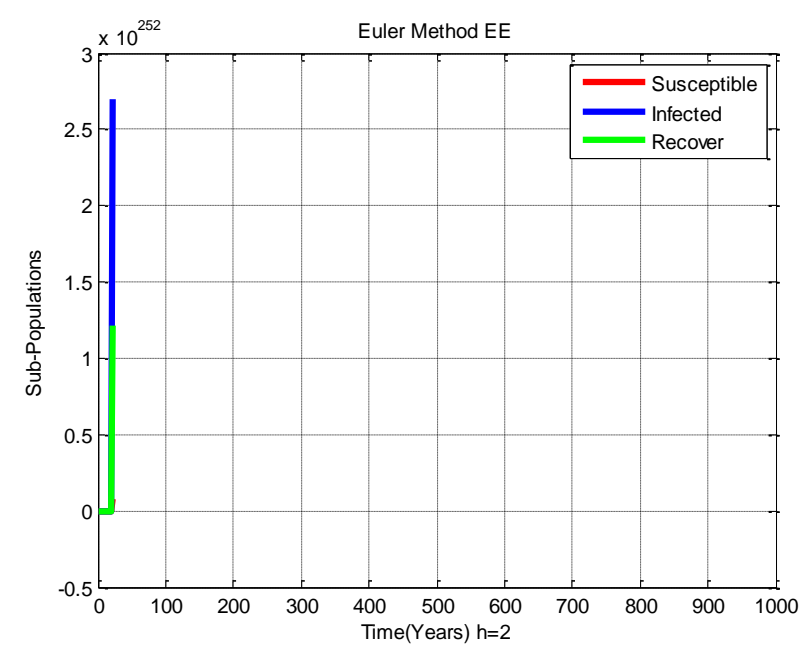

Fig.5 Euler Method (EE), $h=2$

\section{C: Fourth Order Runge-Kutta Scheme}

For Stage-1

$K_{1}=h\left[\lambda-d_{1}-\beta v x^{n}\right]$

$l_{1}=h\left[\beta v x^{n}-a y^{n}\right]$

$m_{1}=h\left[k y^{n}-u v^{n}\right]$

For Stage-2

$$
\begin{aligned}
& k_{2}=h\left[\lambda-d_{1}-\beta v\left(x^{n}+\frac{k_{1}}{2}\right)\right] \\
& l_{2}=h\left[\beta v\left(x^{n}+\frac{k_{1}}{2}\right)-a\left(y^{n}+\frac{l_{1}}{2}\right)\right]
\end{aligned}
$$




$$
m_{2}=h\left[k\left(y^{n}+\frac{l_{1}}{2}\right)-u\left(v^{n}+\frac{m_{1}}{2}\right)\right]
$$

For Stage-3

$$
\begin{aligned}
& k_{3}=h\left[\lambda-d_{1}-\beta v\left(x^{n}+\frac{k_{2}}{2}\right)\right] \\
& l_{3}=h\left[\beta v\left(x^{n}+\frac{k_{2}}{2}\right)-a\left(y^{n}+\frac{l_{2}}{2}\right)\right] \\
& m_{3}=h\left[k\left(y^{n}+\frac{l_{2}}{2}\right)-u\left(v^{n}+\frac{m_{2}}{2}\right)\right]
\end{aligned}
$$

For Stage-4

$$
\begin{aligned}
& k_{4}=h\left[\lambda-d_{1}-\beta v\left(x^{n}+\frac{k_{2}}{2}\right)\right] \\
& l_{4}=h\left[\beta v\left(x^{n}+\frac{k_{2}}{2}\right)-a\left(y^{n}+\frac{l_{2}}{2}\right)\right] \\
& m_{4}=h\left[k\left(y^{n}+\frac{l_{2}}{2}\right)-u\left(v^{n}+\frac{m_{2}}{2}\right)\right]
\end{aligned}
$$

Finally

$$
\begin{aligned}
& x^{n+1}=x^{n}+\frac{1}{6}\left[K_{1}+2 K_{2}+2 K_{3}+K_{4}\right] \\
& y^{n+1}=y^{n}+\frac{1}{6}\left[l_{1}+2 l_{2}+2 l_{3}+l_{4}\right] \\
& v^{n+1}=v^{n}+\frac{1}{6}\left[m_{1}+2 m_{2}+2 m_{3}+m_{4}\right]
\end{aligned}
$$

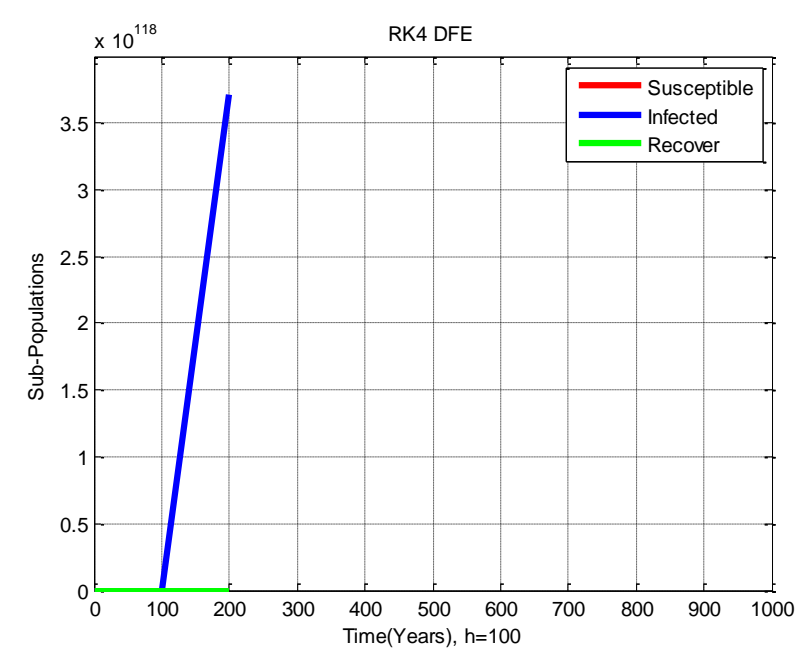

Fig.6 RK-4 Method (DFE), $h=100$ 


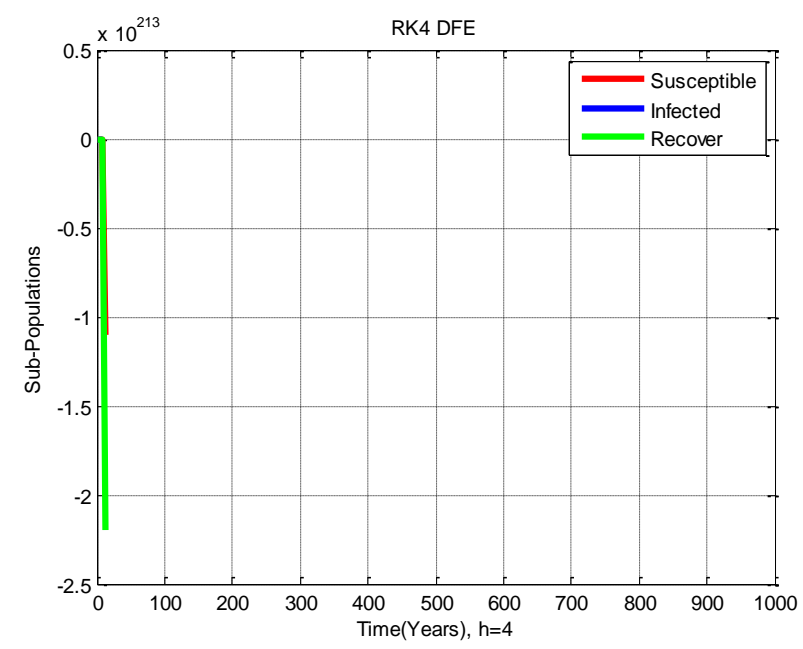

Fig.7 RK-4 Method (DFE), $h=4$

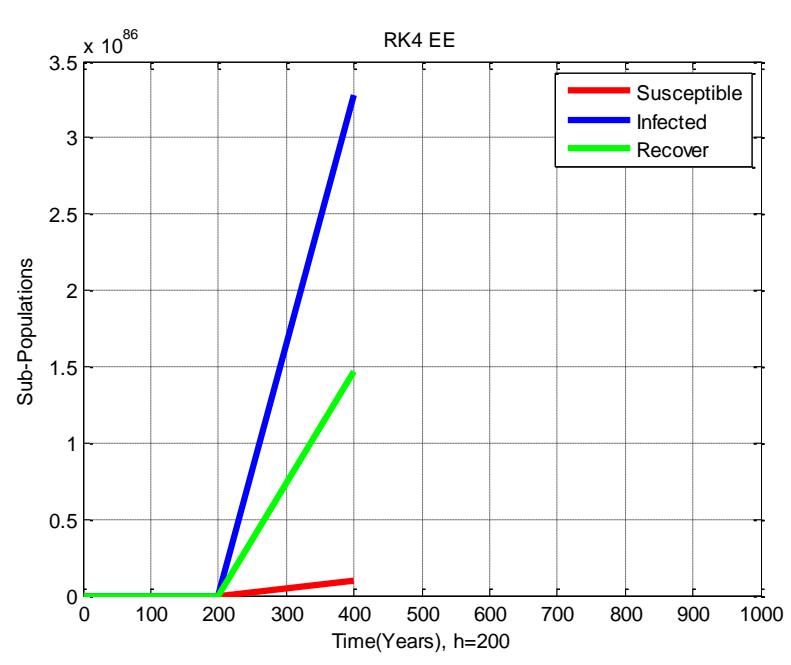

Fig.8 RK-4 Method (EE), $h=200$ 


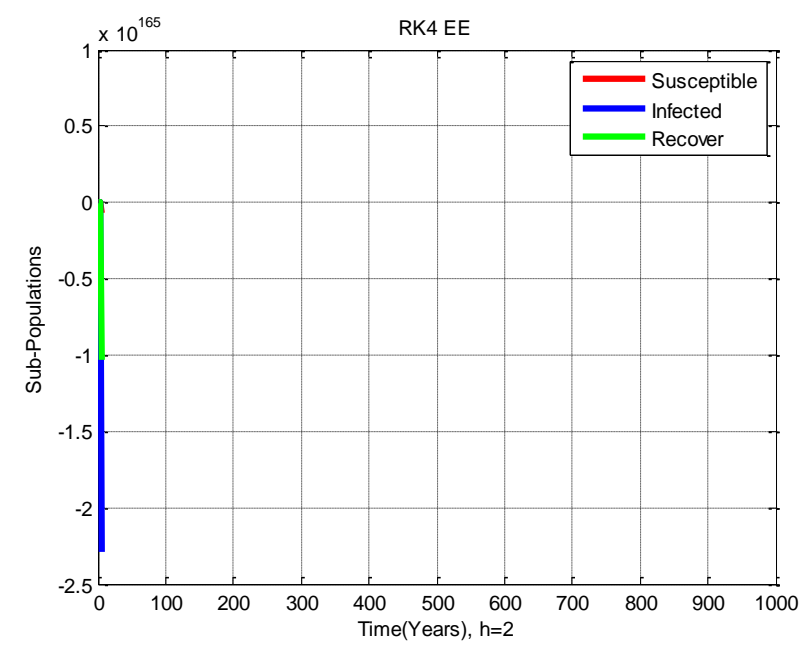

Fig.9 RK-4 Method (EE), $h=2$

\section{D: Non-standard Finite DIFFERENCE MODEL}

Now we show an unreservedly convergent non-standard finite difference(NSFD) numerical model which be there describe on non-standard finite difference modeling concept introduced by Micken's [3]. Now show the covergenence scrutiny of the suggested structure.The NSFD model for the incessant dynamical system is given by:

$$
\begin{aligned}
x^{n+1} & =\frac{x^{n}+h \lambda}{1+h d_{1}+h \beta v^{n}} \\
y^{n+1} & =\frac{y^{n}+h \beta v^{n} x^{n}}{1+h a} \\
v^{n+1} & =\frac{v^{n}+h k y^{n}}{1+h u}
\end{aligned}
$$

\section{A: Convergence Analysis of NSFD Scheme}

Let us define

$$
\begin{aligned}
& E=\frac{x+h \lambda}{1+h d_{1}+h \beta v} \\
& F=\frac{y+h \beta v x}{1+h a} \\
& G=\frac{v+h k y}{1+h u}
\end{aligned}
$$

Now the Jacobian Matrix is given by

$$
J=\left[\begin{array}{lll}
\frac{\partial E}{\partial x} & \frac{\partial E}{\partial y} & \frac{\partial E}{\partial v} \\
\frac{\partial F}{\partial x} & \frac{\partial F}{\partial y} & \frac{\partial F}{\partial v} \\
\frac{\partial G}{\partial x} & \frac{\partial G}{\partial y} & \frac{\partial G}{\partial v}
\end{array}\right]
$$

At DiseaseFree Equilibrium $\varepsilon_{1}=\left(\frac{\lambda}{d_{1}+\beta v}, 0,0\right)$

At Endemic Equilibrium $\varepsilon_{1}=\left(\frac{\lambda}{d_{1}+\beta v}, \frac{\beta v \lambda}{a\left(d_{1}+\beta \lambda\right)}, \frac{k \beta v \lambda}{a u\left(d_{1}+\beta \lambda\right)}\right)$ 
$J^{*}\left(\mathcal{E}_{1}\right)=\left[\begin{array}{ccc}\frac{1}{1+h d_{1}+h \beta v} & 0 & 0 \\ \frac{h \beta v}{1+h a} & \frac{1}{1+h a} & \frac{h \beta x}{1+h a} \\ 0 & \frac{h k}{1+h u} & \frac{1}{1+h u}\end{array}\right]$

There are the following eigen values of above jacobian matrix is:

$\lambda_{1}=\frac{1}{1+h d_{1}+h \beta v}<1$

$\lambda_{2}=\frac{1}{1+h a}<1$

$\lambda_{3}=\frac{1}{1+h u}<1$

\section{Lemma3.1 [12]}

For the quadratic equation $\lambda^{2}-A \lambda+B=0,\left|\lambda_{i}\right|<1, i=1,2$; iff the following conditions are satisfied:

(1) $1-A+B>0$

(2) $1+A+B>0$

(3) $B<1$

\section{B: Numerical Experiments}

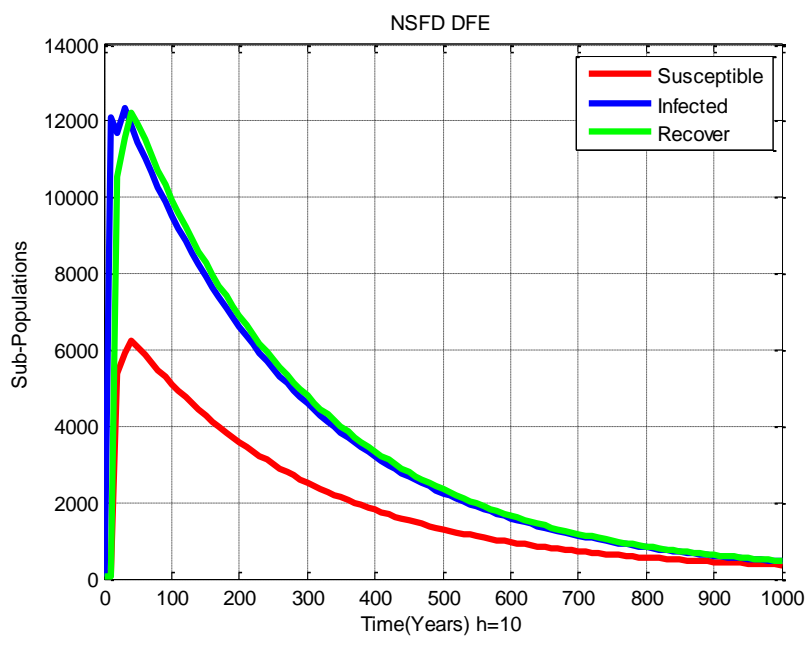

Fig.10 NSFD Method (DFE), $h=10$ 


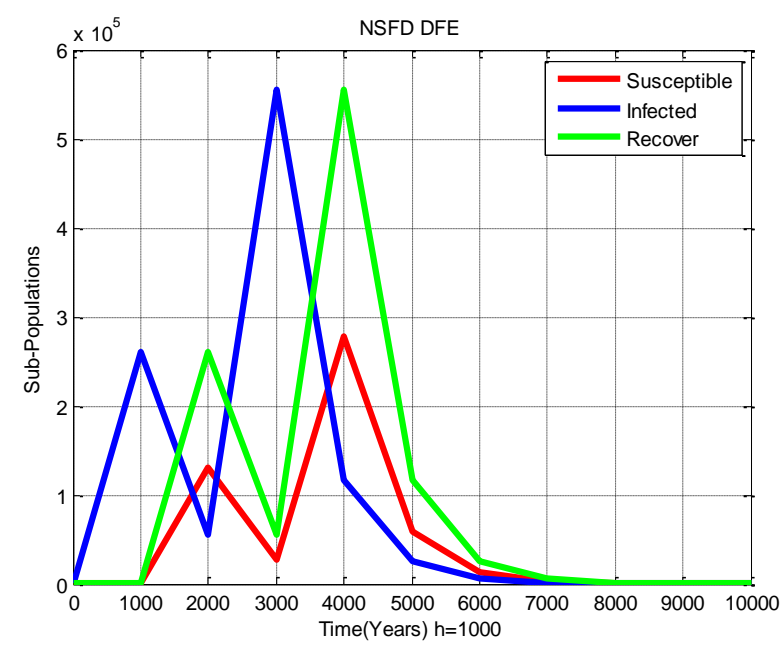

Fig.11 NSFD Method (DFE), $h=1000$

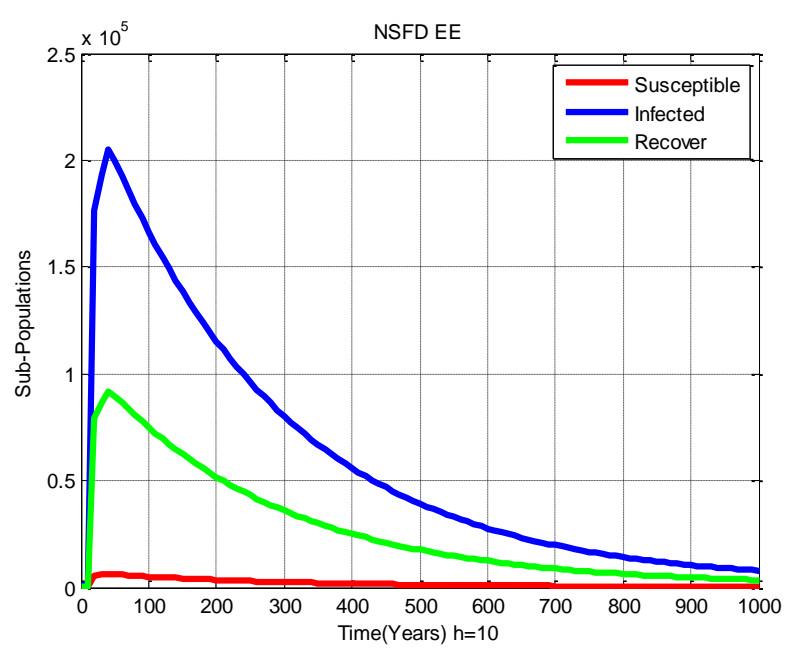

Fig.12 NSFD Method (EE), $h=10$

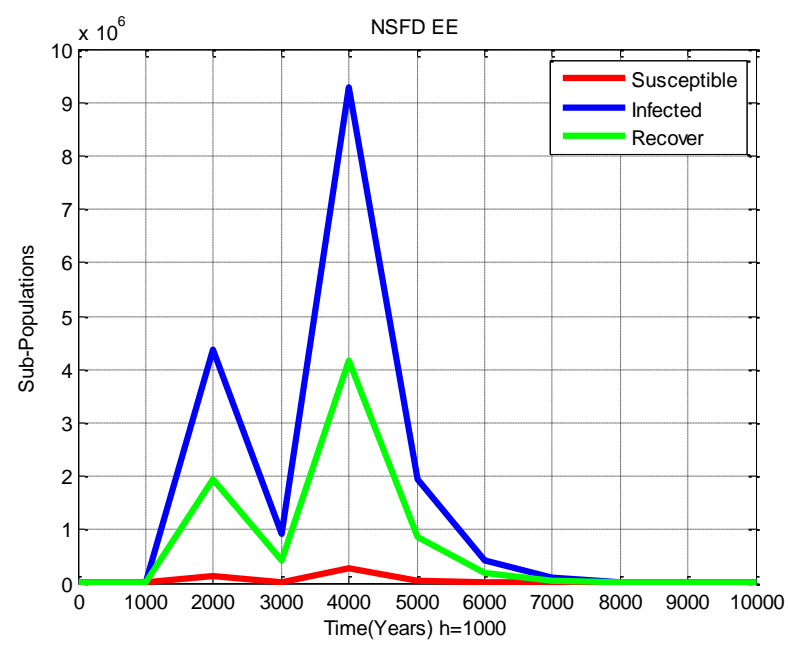

Fig.13 NSFD Method (EE), $h=1000$ 


\section{C: Comparison Analysis}

In this section, we see the comparison among of two standard difference schemes and non-standard difference scheme in epidemiology

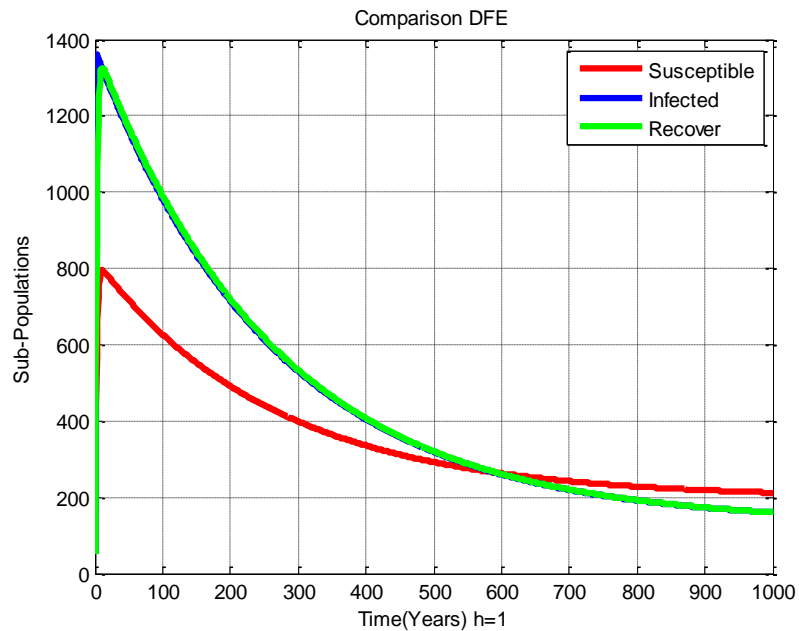

Fig.14 comparison $(\mathrm{DFE}), \mathrm{h}=1$

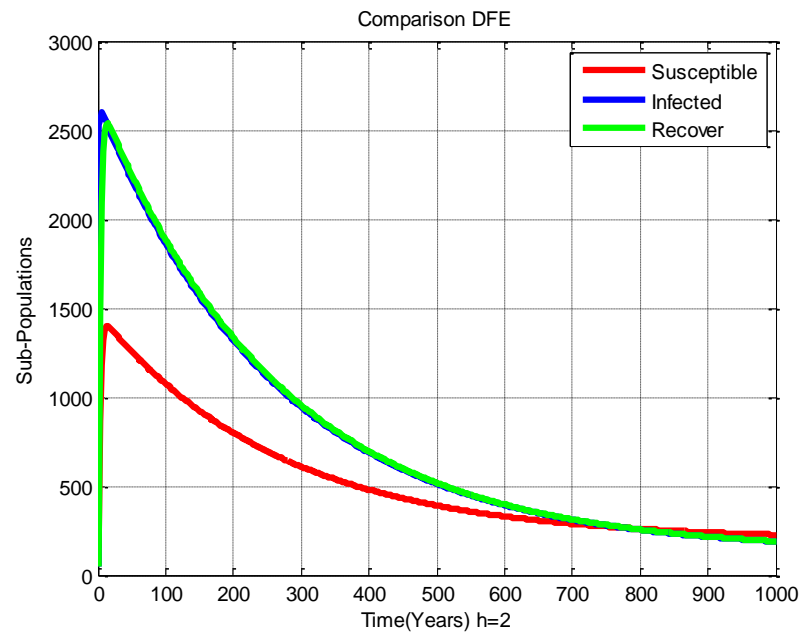

Fig.15 comparison (DFE), $h=2$ 


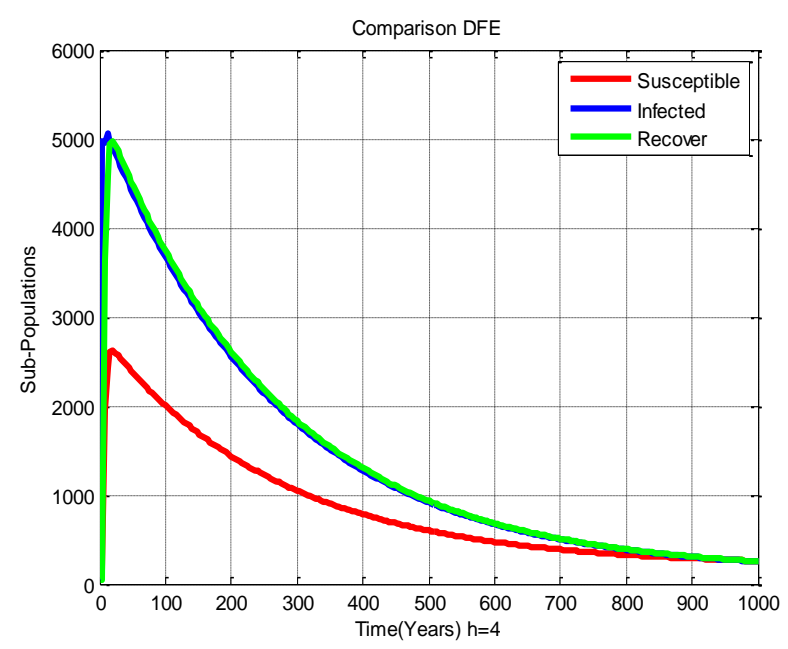

Fig.16 comparison (DFE), $h=4$

\section{Results and Discussion}

The model of transmission dynamics of Hepatitis B virus disease consumes introduced expending PSIT Model. (i.e Threatened, Susceptible, Infected and Treated). The constancy of solid positions i.e the Disease free equilibrium(DFE) and Endemic equilibrium facts(EE) deliberated numerically. We describe an unqualifiedly constant Non-Standard Finite Difference (NSFD) structure aimed at the incessant dynamical system. The suggested structure exists dynamical consistant, numerically steady and holds all the athentic assets of the incessant model. The outcomes equaled well known standard finite difference schemes i.e Euler's and Runge-Kutta method of order 4 (RK-4). The Euler and RK-4 are provisionally convergent and diverge of the assured ethics of step size ' $h$ ' while the constructed NSFD scheme for every assessment used to residues convergent.

\section{References}

1. World Health Organization. Hepatitis B[OL], March 2015, http://www.who.int/mediacentre/factsheets/fs204/en/.

2. Y. Muroya, Y. Nakata, G. Izzo, et al., Permanence and global stability of a class of discrete epidemic models, Nonlinear Analysis: Real World Applications 12 (2011), 2015-2117.

3. Y. Zheng, L. Min, Y. Ji, et al., Global Stability of Endemic Equilibrium Point of Basic Virus Infection Model with Application to HBV Infection, Journal of Systems Science and Complexity, 23 (2010), 1221-1230.

4. L. Min, Y. Su and Y. Kuang, Mathematical analysis of a basic model of virus infection with application to HBV infection, Rocky Mountain Journal of Mathematical 38 (2008), 1573-1584.

5. S.A. Gourley, Y. Kuang and J.D. Nagy, Dynamics of a delay differential model of hepatitis B virus, Journal of Biological Dynamics 2 (2008), 140-153.

6. J. M. Murray, R. H. Purcell and S. F. Wieland, The half-life of hepatitis B virions, Hepatology 44 (2006), 1117-1121.

7. D. Zhang and B. Shi, Oscillation and global asymptotic stability in a discrete epidemic model, Journal of Mathematical Analysis and Applications 278 (2003), 194-202. 
8. L. G. Guidotti, R. Rochford, J. Chung, et al., Viral clearance without destruction of infected cells during acute HBV infection, Science 284 (1999), 825-829.

9. M.A. Nowak, S. Bonhoeffer, A.M. Hill, et al., Viral dynamics in hepatitis B virus infection, Proceedings of the National Academy of Sciences 93 (1996), 4398-4402.

10. L. J. S. Allen, Some discrete-time SI, SIR, and SIS epidemic models, Journal of Mathematical Biology 124 (1994), 83-105. 